\title{
Gesture mimicry in expression of laughter
}

\author{
Harry Griffin*, Giovanna Varni ${ }^{\dagger}$, Gualtiero Volpe ${ }^{\ddagger}$ \\ Gisela Tomé Lourido*, Maurizio Mancini ${ }^{\ddagger}$ and Nadia Bianchi-Berthouze* \\ * UCL Interaction Centre, University College London, United Kingdom \\ Email: \{harry.griffin; gisela.tome; nadia.berthouze\}@ucl.ac.uk \\ ${ }^{\dagger}$ Institut des Systèmes Intelligents et de Robotique, Université Pierre et Marie Curie - Paris 6, CNRS UMR 7222, France \\ Email: varni@isir.upmc.fr \\ $\ddagger$ Casa Paganini - InfoMus, DIBRIS - University of Genoa, Italy \\ Email: \{gualtiero.volpe; maurizio.mancini\}@unige.it
}

\begin{abstract}
Mimicry and laughter are two social signals displaying affiliation among people. To date, however, their relationship remains uninvestigated and relatively unexploited in designing the behaviour of robots and virtual characters. This paper presents an experiment aimed at examining how laughter and mimicry are related. The hypothesis is that hand movements a person produces during a laughter episode are mimicked through equivalent or other hand movements other participants in the interaction produce when they laugh. To investigate this, we analysed mimicry at two levels of specificity during laughter and non-laughter periods in a playful triadic social interaction. Changes in mimicry rates over the whole interaction were analysed as well as possible leader-follower relationships. Results show that hand movement rates were varied and strongly dependent on group. Even though hand movement are more frequent during laughter, mimicry does not increase. Mimicry levels, however, increase over the course of a session indicating that familiarity and comfort may increase emotional contagion.
\end{abstract}

Keywords-mimicry; gesture; laughter; social interaction

\section{INTRODUCTION}

Mimicry is an important facet of interaction as it displays affiliation among people. Various forms of mimicry have been found and studied in social interaction [1][2][3] and in interaction with computer avatars [4][5]. Laughter is another strong signal of a mutually understood affiliative state that is able to facilitate sociability and cooperation [6], and so it could be seen as overlapping with mimicry in its social function. At present, however, the relationship between mimicry and laughter is relatively uninvestigated.

This paper presents an experiment for the analysis of gesture mimicry during laughter, aimed at exploring how laughter affects mimicry e.g., whether gestures are more frequently mimicked during laughter. Such gestures in laughter have been noted in historical descriptions "in the height of the laugh ... some plant the elbows on the knees; ... the hands are thrown into the air or clapped on the thighs; ... the limbs jerk; ... the fists pound; ... the hand is placed over the eyes, mouth, or both; ..." [7]. This study focusses on hand gestures only, which are peripheral to the core production of laughter, but may correlate with laughter due to being cued by an exhilarated, amused state or replacing speech information that it is not possible to produce during laughter. Gestures are defined for the purposes of this paper as any hand movement and do not have to have a specific meaning associated with them. To test this, groups of 3 friends took part in a semi-structured session, which used a word game to generate pseudo-conversational interactions over a video-conferencing link. Gestures were annotated from recordings of these interactions in terms of laterality, type and direction. Gesture rates across participants and groups in laughter vs. non-laughter periods were examined to give context for mimicry analysis. We used two definitions of mimicry that allow for different degrees of gesture similarity. Under these definitions, several facets of mimicry were then analysed: (i) development of mimicry over the whole session; (ii) mimicry during laughter vs. non-laughter periods; (iii) leader-follower relationships of mimicry between dyads in laughter vs. non-laughter periods. Inclusion and control of mimicry and laughter in virtual characters will provide benefits in terms of perceived naturalness of behaviour and contribute towards maximising efficacy in human-avatar interactions.

The remainder of the paper is organised as follows: Section II introduces the background this study is grounded on; Section III describes the experimental scenario and setup; Section IV provides a description of the adopted methodology; Section V focusses on the analysis and Section VI on results.

\section{BACKGROUND}

Mimicry - "the tendency to imitate facially, vocally or posturally people with whom we are interacting" [8] - is thought to be an automatic unconscious process that serves an affiliative role by promoting liking [9]. This function of mimicry is supported by the finding that its level is dependent on the social context and the relationship between interlocutors. Lanzetta and Englis [10] showed that the emotional response of observers to facial expressions was partly influenced by their expectation of the nature of the interaction (cooperative or competitive). Furthermore an affiliation goal of which participants are not conscious augments nonconsicous mimicry [11]. Although happy facial expressions from ingroup and outgroup members are mimicked equally, anger and sadness expressions are mimicked significantly more when seen on an ingroup than an outgroup member [8].

Laughter is another signal having affiliative function, for example it is returned so as to signal a mutually understood affiliative state. Findings in [8] support the hypothesis that purely positive, Duchenne laughter will elicit mimicry of some 
form regardless of its origin. Laughter with mixed emotional content (e.g., Schadenfreude), or negative emotional content (e.g., mocking), may elicit mimicry only when it is produced by an ingroup member and the observer shares these emotions. Not all actions that can be mimicked have the same inherent emotional signalling strength. For example, facial expressions that may occur during laughter signal a specific emotion, but gestures that accompany laughter may not have a clear emotional valence. As such, laughter mimicry may not occur across all possible channels and, within a single channel, some actions may be more likely to elicit mimicry than others.

The simplest form of laughter-related mimicry is the return of laughter i.e., the listener laughing in response to the speaker laughing. Laughter return is an inherent part of conversation. Laughter is appended to the end of a statement to disambiguate the preceding statement or invite laughter from an interlocutor. Note that sequential laughter does not necessarily imply laughter contagion as the two laughs may be prompted by a mutually experienced external event.

Even if the laughter trigger is a mutually observed event or shared thought, mimicry can occur through similarity between the laughs of two or more people. More subtle and fine grained types of laughter mimicry may include similarities in the body movements of two people when they are laughing [12]. Numerous studies have shown that interlocutors mimic each other's postures [13], repeat each other's gestures [9][14] and coordinate postural sway [15].

\section{EXPERIMENTAL SCENARIO AND SETUP}

\section{A. Scenario}

The experimental scenario involved 3 participants at a time, playing the yes/no game via a videoconferencing system. In the game two participants asked questions to which the third participant (answerer) was obliged to respond promptly without using the words "yes", "no" or any derivative thereof. If the answerers avoided saying the prohibited words for 2 minutes they won the game round; otherwise they lost that round. This scenario was chosen in order to naturally and easily elicit laughter among the participants.

\section{B. Setup and Sensors}

For each session, 3 participants sat in 3 separate rooms. They could communicate only via Oovoo video-conferencing software $^{1}$, which provided voice communication via a headset, and video communication via images of the other 2 participants on a single display. The video-conferencing latency was tested by comparing direct and video-link communication and found to be very short. No participants commented on having any difficulty identifying facial expressions. Participants' actions were monitored with (i) a Kinect sensor (Kinect for Windows, v1.8) capturing video, depth map, action-unit, and face tracking and (ii) a rgb webcam (25fps, 640x480). To track body movements, green markers were fixed to shoulders. Kinect and body marker data were collected for future analyses. Voices were recorded with a Sennheiser PC 230 headset.

\footnotetext{
${ }^{1}$ www.oovoo.com
}

\section{Methods}

\section{A. Participants}

Groups of friends were recruited via advertisement. 7 groups of 3 participants completed the recording session (mean age $=$ 24.4, SD = 4.75). 3 groups were all-female, 2 groups had 1 male and 2 female participants, 2 groups had 1 female and 2 male participants. Two participants were left-handed. Participants were reimbursed for their time at a fixed rate.

\section{B. Procedure}

The scenario was explained to the participants and informed consent obtained. Participants were not told that the recordings were designed to elicit laughter; instead the experiment was presented as an investigation of conversational interaction. The participants completed the STCI-T-60 [16], TIPI [17], and PhoPhiKat-45 [18], and were then seated in separate rooms. For each game round, one participant took the role of answerer and the other two took the role of questioners. Participants took turns in the answerer role and at least 9 rounds were undertaken per group, 3 rounds of each participant taking the role of answerer. More rounds were added at the experimenter's discretion if the rounds were very short i.e., the round was lost within a few seconds. All the participants' behaviour was recorded and analysed including periods between game rounds in which they were encouraged to chat. The scenario led to largely natural conversational behaviour.

This work focusses only on 4 groups of 3 participants that at the time of analysis had been annotated for laughter (2 allfemale groups, 2 groups with 2 male and 1 female, mean age of these 12 participants was $24.5 \mathrm{y}, \mathrm{SD}=6.01$ ). The mean session duration for these groups was 23 minutes 6 seconds (minimum 22 minutes 16 seconds, maximum 23 minutes 37 seconds). Laughter was segmented at two levels (Visual and Auditory) with the aim of being over-inclusive in annotation of laughter. The goal was to get four timestamps typically in the order Visual Onset - Auditory Onset - Auditory Offset Visual Offset, with the auditory laugh almost always contained within a longer visual laugh. For the purposes of this study the laughter annotation used is that derived from video, rather than audio, since this parallels the visual annotation of hand gestures. The mean number of laughs per participant was 119.8 $(\mathrm{SD}=95.7, \min =33, \max =378)$; the mean duration of laughter, computed as the mean of all laughs, was $2.84 \mathrm{~s}$ (SD = 1.13). The average duration of the session that each participant spent laughing was $20.9 \%(\mathrm{SD}=11.4 \%$, $\min =4.12 \%$, $\max$ $=40.7 \%$ ).

\section{Gesture Annotation and Inter-rater Reliability}

Hand gestures were annotated according to a scheme developed by the authors and described below. The gesture taxonomy was developed to be a mid-level description indicative, at a coarse level, of the purpose/meaning of the gesture, while being relatively simple to apply. Gestures were annotated in Elan $^{2}$ for Laterality, Type and Direction as described in Table

\footnotetext{
${ }^{2}$ https://tla.mpi.nl/tools/tla-tools/elan/
} 
I. As for Type, we wanted to differentiate between pointing, clapping, and illustrator. When gestures did not belong to any of these categories, they were labelled as "other" e.g., crossing the arms. All gestures were annotated for all categories e.g., both hands pointing forward was B_pointing_forward, placing the right hand on the mouth was R_other_head. A primary rater annotated gestures of the 12 participants (3 participants $\mathrm{x} 4$ groups) and 2 ancillary raters annotated the same 5 participants from different groups. The annotations were manually aligned to maximise overlap between raters and the level of agreement between each ancillary rater and the primary rater calculated. There was considered to be agreement between two raters for a given gesture if the temporal window reported by the two raters overlapped. Lack of agreement therefore occurred when one rater reported a gesture for which there was no temporally overlapping gesture reported by the other rater. Most disagreements between raters concerned gesture Type and Direction. As for Type, disagreement was related to the identification of illustrators. Concerning Direction, there was confusion between "forward" and "trunk". Percentage agreement was calculated relative to the number of gestures identified by the rater who identified more gestures. For example, if rater 1 identified 100 gestures and rater 2 identified 87 gestures, of which 84 overlapped in time with the gestures identified by rater 1 , the agreement for the presence of gestures would be $84 \%$. Percentage agreement for Laterality, Type and Direction were also computed in the same way. Agreement for the presence of gestures was $85.6 \%(\mathrm{SD}=9 \%)$; agreement for gesture laterality was $81.9 \%$ ( $\mathrm{SD}=10 \%)$; agreement for gesture type was $79.3 \%$ ( $\mathrm{SD}=9.4 \%$ ); agreement for gesture direction was $76.1 \%(\mathrm{SD}=11.1 \%)$. These levels of agreement were considered sufficiently high to use the primary rater's annotation for all participants for all subsequent analyses.

\section{General and Specific Mimicry}

Analyses of mimicry (also termed "convergence" and "synchrony") in the literature, vary from matching of specific gestures [14] to much broader categorisations that allow any body movements within a given time window to be considered as mimicry [19]. In order to take account of this broad range of definitions we considered mimicry at both a specific and a more general level:

- Specific mimicry. It was here defined as an exact matching of a hand gesture within $3 \mathrm{~s}$ of the initial gesture e.g., participant 1 covers her mouth then participant 2 covers her mouth; and

- General mimicry. It was here defined as any hand gesture occurring within $3 \mathrm{~s}$ of the initial gesture e.g., participant 1 covers her mouth then participant 2 points forward.

To achieve identical analyses using these two different types of mimicry, a gesture coding system was developed to allow the data to be readily converted to a format suitable for each specific analysis. Using this system the gesture annotation data was coded in two different ways. For general mimicry all gestures were given the same code. For specific mimicry each gesture was given a unique code resulting from the sum of 3
TABLE I

GESTURE CODING: UNIQUE GESTURE ARE REPRESENTED AS THE SUM OF VALUES FOR LATERALITY, TYPE, AND DIRECTION.

\begin{tabular}{|c|c|c|}
\hline Laterality Code & Type Code & Direction Code \\
\hline Both: 100 & Clapping: 10 & Forward: 1 \\
\hline Left: 200 & Illustrator: 20 & Head: 2 \\
\hline Right: 200 & Other: 30 & Lateral: 3 \\
\cline { 2 - 3 } & Pointing: 40 & Trunk: 4 \\
\hline
\end{tabular}

integer values for Laterality, Type, and Direction of gesture as shown in Table I. For example, a forward pointing gesture performed by a participant with the right hand is codified as: 241 , that is right(200)+ pointing $(40)+$ forward(1). Note that the left vs. right distinction was removed so gestures can now be considered as unilateral vs. bilateral. This simplification was made because the full-face nature of the remote interaction renders any distinction between left and right meaningless. In a co-localised setting in which participants are sat to each others' left or right, the distinction would be meaningful. Further, for each participant a unique non-gesture code was defined. Using a unique non-gesture code for each participant prevented periods of non-gesturing from being labelled as mimicry in the analysis. The unique code was defined as follows: $300+(\text { group number })^{3}+$ participant number. An additional recoding allowed comparison of mimicry during laughter-only $(L O)$ and non-laughter only $(N L O)$ periods within the session. This recoding allocated unique codes for whichever periods (laughter or non-laughter) were being excluded, which prevented any gestures from being taken into account for analysis during those periods. The effect of this recoding was to define mimicry as possible only when both participants were in the same state i.e., both laughing or both not-laughing. Starting from these coded data, time series were synthesised with a sampling rate of $100 \mathrm{~ms}$.

\section{ANALYSIS}

To analyse the data, Cross-Recurrence Quantification Analysis $(C R Q A)$ was applied. In recent years, CRQA has rapidly gained attention in the behavioural sciences. A particular focus has been on measuring how and to what extent dyads exhibit couplings of features in time, especially in conversational tasks (e.g., [20][21][22][14]). CRQA is the bivariate extension of recurrence allowing measurement of dependencies between two continuous/categorical time series describing two dynamical systems in a feature space [23]. This analysis explores and focusses on co-visitation patterns of these time series in the feature space, that is, it looks for those times when a value of the time series of the first system recurs in the other system. As in recurrence, cross-recurrence was firstly introduced through a plot: the Cross-Recurrence Plot (CRP) is a rectangular area spanned by the two (discretised or quantised) time series where each black point corresponds to the times the two systems roughly co-visit the same area in feature space. The oriented diagonal lines are the most interesting pattern in a CRP, representing segments of time for which the two systems run parallel although with a certain relative delay. Using 
categorical data requires some arrangements of the original analysis. For example, the threshold to claim recurrence is set to zero so that only exact matches of the same category will be considered as recurrent and each time series needs a unique value to be assigned to all its samples that do not belong to any of the defined categories. A more detailed description of CRQA applied to categorical data is available in [24][25]. The most relevant measure in CRQA is the diagonal-wise crossRecurrence Rate $(R R)$ revealing the probability of occurrence of similar features values in both systems at a given relative delay $\tau$. It expresses the density of recurrent points in a plot over time distances from the main diagonal line in a CRP (called Line Of Coincidence, $L O C$ ). Basically, it shows how many times (identified by the number of recurrent points) the two systems exhibit similar features values at the same time. $\mathrm{RR}$ is computed as:

$$
R R_{\tau}=\frac{1}{N-\tau} \sum_{l=1}^{N-\tau} l P(l)_{\tau}
$$

where $P(l)_{\tau}$ is the number of diagonal lines of length $l$ on each diagonal parallel to the LOC. Positive and negative delays are represented in respect to the $\operatorname{LOC}(\tau=0)$ by positive (above the LOC) and negative (below the LOC) $\tau$, respectively. Diagonal-wise cross-recurrence rate measures were computed on the time series on participant pairs within each group. The CRQA analysis presented in this work was carried out by using the $R$ package crqa developed by Coco and colleagues [25].

\section{A. Development of Mimicry During Session}

The first interest in this study was to evaluate how the recurrence rate evolves over the time course of the entire session. It was expected that this rate would increase over time as participants become more comfortable and confident in the interaction scenario and their communication and interaction improves as a result. This would reflect mimicry as a form of communication [26] and a social glue [9]. The time series were windowed and for each of the resulting (overlapped) windows the cross-recurrence plot was built and the crossrecurrence rate was computed over a range of time delays $\tau$. Then, the average of these rates in each window was taken as representative value of the recurrence in that window. Finally, the averaged cross-recurrence rate profile was computed.

The choice of the window size and of the overlapping was critical and is the result of the trade-off between the nature of the mimicry (a phenomenon with specific timing) and the statistical nature of CRQA. According to previous analyses [19], a window size of $6 \mathrm{~s}$ with an overlap of $3 \mathrm{~s}$ was chosen. In this way, all the possible mimicry episodes are accounted in the analysis and a reasonable number of samples are available for CRQA. The $6 \mathrm{~s}$ window allows gestures occurring within $+/-3 \mathrm{~s}$ of each other to be considered as mimicry.

\section{B. Mimicry During Laughter vs Non-laughter}

It was inevitable that windows on which the RR was computed would include a varying percentage of laughter and nonlaughter samples. From annotation it emerged that there are laughter episodes shorter/longer than the window size of $6 \mathrm{~s}$. For example a window containing a 2 s laugh will contain nonlaughter periods as well. This affects the interpretation of the results both from a visual and a computational point of view. This problem was tackled by defining two metrics that allowed us to normalise the amplitude of the peaks in the RR profile according to the number of laughter and non-laughter windows for which RR values greater than zero were possible. Such windows were identified by laughter from both participants or non-laughter from both participants, respectively, occurring within $3 \mathrm{~s}$ of each other. The first metric is the mean RR in such LO and NLO windows. The second is the percentage of windows in which an LO and NLO RR $>0$ was possible that actually showed a $\mathrm{RR}>0$.

\section{Leader-Follower Relationship in Mimicry}

The computation of cross-recurrence rate also allows us to detect which of the 2 participants in each pair is mimicked (leader) by the other one (follower), if at all. That is which of the 2 participants of each pair tended on average to gesture first in gesture-gesture pairings that are recognised by the cross recurrence analysis as mimicry. Obviously, this gestural mimicry relationship has to be understood with reference to the above-given mimicry definitions. For each pair of participants, the CRPs were built for laughter and non-laughter periods for both specific mimicry and general mimicry. Then, the RR was computed for $\tau$ in the range $[-3 s, 3 s]$. Positive delays refer to delays of the second time-series in respect to the first one, negative delays refer to delays of the first time-series in respect to the second one. That is, these delays show the relative time instants at which one participant gestures behind the other one. This allows us to identify who is leading who in the pair. These leader-follower patterns are reflected in an asymmetrical shape (right/left skewness) of the RR profile. This approach was already used in [14]. To quantify such an asymmetrical shape, the areas under the RR curves (AUCs RR) in the ranges $[-\tau, 0]$ and $[0, \tau]$ were taken as a quantitative measure of the extent to which the participants lead and follow each other.

\section{RESULTS}

\section{A. Gesture Frequency}

A broad assessment of gestures (independent of mimicry) was carried out to elucidate overall patterns of behaviours that might influence patterns of mimicry. To this end, gestures were labelled according to whether they began during a laugh or non-laugh period, according to the visual laughter annotation. Three major observations can be made from the data as shown in Figure 1 and the results of the personality questionnaires:

- Gesture rates increase during laughter. The mean gesture rate of the 12 participants in this analysis was 2.70 gestures/minute $(\mathrm{SD}=2.05)$ during non-laughter and 4.38 gestures/minute $(\mathrm{SD}=3.08)$ during laughter. The paired 2 -tailed t-test revealed statistical significant difference in the use of gestures between the two situations $(p<.02)$. This is true of all but 2 participants (group 3 participants 1 and 2). In the absence of any other factors, mimicry 


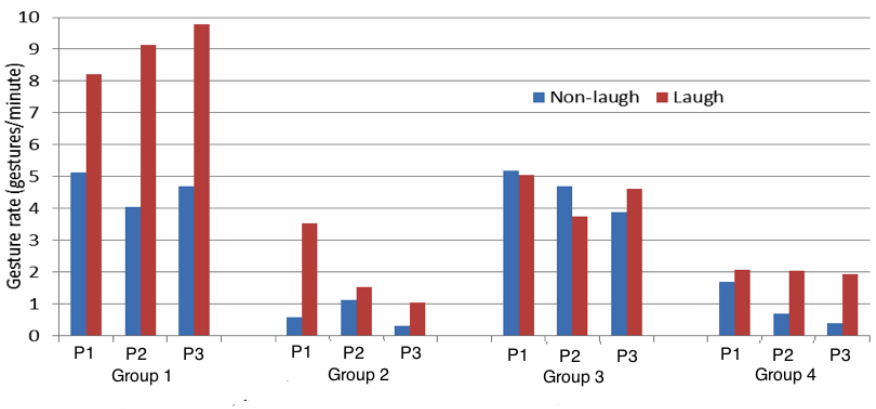

Fig. 1. Gestures rates for the 4 groups of participants

during laughter might therefore be expected to increase during laughter relative to non-laughter. Participants' gesture rates during laughter are correlated with gesture rates during non-laughter $\left(r^{2}=0.608, p<.003,2\right.$ tailed) suggesting that individual differences in amount of gesturing persist from non-laughter to laughter situations.

- Gesture rate is strongly influenced by group i.e., participants in the same group have similar gesture rates. One-way ANOVAs with participants' group as the independent variable return $F(3,11)=43.1, p<.0001$ for gesture rate during non-laughter and $F(3,11)=46.7$, $p<.0001$ for gesture rate during laughter. This effect is not reflected by similar participant personalities within groups: of the 11 components of the TIPI, STCI-T-60 and PhoPhiKat-45 there is an effect of group only on TIPI-Conscientiousness $(F(3,11)=9.18, p<.006)$ and STCI-T-60-Seriousness $(F(3,11)=3.22, p<.009)$ and neither of these are significant $(p<.0045)$ after correction for multiple comparisons.

- Gesture rates are very low in groups 2 and 4. The gesture rate is so low for these groups that hand-gesture mimicry may be too infrequent to meaningfully analyse.

- Additionally, neither gesture frequency during laughter nor during non-laughter were correlated with any component of TIPI, STCI-T-60, or PhoPhiKat-45 (all correlations $p>.07$ before correction for multiple comparisons).

\section{B. Development of Mimicry During Session}

Table II shows the mean RR for all participant pairings as calculated for the first and second half of each session. All the values under $0.1 \%$ are set to zero. Specific mimicry increased in the second half of the session (paired t-test, 2tailed, $p<.05$ ); however, there was no effect for general mimicry $(p=.4)$. The low RR for groups 2 and 4 in both specific and general mimicry reflect the low rates of gesturing in these groups. These results, along with the low number of gestures annotated for these groups, mean that further analysis of groups 2 and 4 would not be productive. Groups 2 and 4 are therefore not analysed further. Several observations can be made on the basis of the data of groups 1 and 3:

- General mimicry RRs are larger than specific mimicry RRs. This is a mathematical inevitability since all specific
TABLE II

AVERAGED RR IN FIRST AND SECOND HALF OF ALL SESSIONS.

\begin{tabular}{|c|c|c|c|c|c|}
\cline { 3 - 6 } \multicolumn{2}{c|}{} & \multicolumn{2}{c|}{ Specific Mimicry } & \multicolumn{2}{c|}{ General Mimicry } \\
\cline { 3 - 6 } \multicolumn{2}{c|}{} & 1st half & 2nd half & 1st half & 2nd half \\
\hline \multirow{3}{*}{ Group 1 1 } & P1-P2 & $0.84 \%$ & $1.80 \%$ & $75.55 \%$ & $54.55 \%$ \\
& P1-P3 & $0.38 \%$ & $0.42 \%$ & $67.91 \%$ & $66.18 \%$ \\
& P2-P3 & $0.22 \%$ & $2.13 \%$ & $69.71 \%$ & $64.89 \%$ \\
\hline \multirow{3}{*}{ Group 2 2 } & P1-P2 & $0 \%$ & $0.22 \%$ & $0 \%$ & $0.40 \%$ \\
& P1-P3 & $0 \%$ & $0 \%$ & $0 \%$ & $0 \%$ \\
& P2-P3 & $0 \%$ & $0 \%$ & $0 \%$ & $0.29 \%$ \\
\hline \multirow{3}{*}{ Group 33 3 3 P1-P2 } & $0.40 \%$ & $0.72 \%$ & $1.08 \%$ & $3.04 \%$ \\
& P1-P3 & $0.12 \%$ & $0.79 \%$ & $1.32 \%$ & $4.33 \%$ \\
& P2-P3 & $0 \%$ & $0.34 \%$ & $0.59 \%$ & $3.37 \%$ \\
\hline \multirow{3}{*}{ Group 4 4 } & P1-P2 & $0 \%$ & $0 \%$ & $0 \%$ & $0.21 \%$ \\
& P1-P3 & $0 \%$ & $0 \%$ & $0 \%$ & $0 \%$ \\
& P2-P3 & $0 \%$ & $0 \%$ & $0 \%$ & $0 \%$ \\
\hline \multicolumn{2}{|c|}{ Mean } & $\mathbf{0 . 1 6 \%}$ & $\mathbf{0 . 5 3 \%}$ & $\mathbf{1 8 . 0 1 \%}$ & $\mathbf{1 6 . 4 4 \%}$ \\
\hline \multicolumn{2}{|c|}{ SD } & $0.26 \%$ & $0.73 \%$ & $32.0 \%$ & $27.57 \%$ \\
\hline
\end{tabular}

TABLE III

SPECIFIC AND GENERAL MIMICRY RR FOR LAUGHTER ONLY (LO) AND NON-LAUGHTER ONLY (NLO).

\begin{tabular}{|c|c|c|c|c|c|}
\hline & \multicolumn{2}{|c|}{ Average RR value } & \multicolumn{2}{|c|}{ \% windows with $R R>0$} \\
\hline & & LO & NLO & LO & NLO \\
\hline \multicolumn{6}{|c|}{ Specific mimicry } \\
\hline & P1-P2 & $1.08 \%$ & $1.10 \%$ & $16.07 \%$ & $5.14 \%$ \\
\hline \multirow[t]{2}{*}{ Group 1} & P1-P3 & $0.30 \%$ & $0.21 \%$ & $6.88 \%$ & $2.56 \%$ \\
\hline & P2-P3 & $0.68 \%$ & $0.73 \%$ & $13.51 \%$ & $5.45 \%$ \\
\hline & P1-P2 & $0.34 \%$ & $0.38 \%$ & $6.42 \%$ & $5.62 \%$ \\
\hline \multirow[t]{2}{*}{ Group 3} & P1-P3 & $0.31 \%$ & $0.06 \%$ & $2.09 \%$ & $2.51 \%$ \\
\hline & P2-P3 & $0 \%$ & $0.13 \%$ & $1.92 \%$ & $3.96 \%$ \\
\hline \multicolumn{2}{|c|}{ Mean } & $0.45 \%$ & $0.44 \%$ & $7.82 \%$ & $3.96 \%$ \\
\hline \multicolumn{2}{|c|}{ SD } & $0.38 \%$ & $0.40 \%$ & $5.85 \%$ & $1.56 \%$ \\
\hline \multicolumn{6}{|c|}{ General mimicry } \\
\hline \multirow{3}{*}{ Group 1} & P1-P2 & $6.45 \%$ & $3.54 \%$ & $34.82 \%$ & $15.94 \%$ \\
\hline & P1-P3 & $1.88 \%$ & $2.45 \%$ & $23.75 \%$ & $14.36 \%$ \\
\hline & P2-P3 & $2.75 \%$ & $2.95 \%$ & $27.93 \%$ & $16.59 \%$ \\
\hline \multirow{3}{*}{ Group 3} & P1-P2 & $0.49 \%$ & $1.44 \%$ & $9.17 \%$ & $12.31 \%$ \\
\hline & P1-P3 & $1.22 \%$ & $1.21 \%$ & $16.75 \%$ & $12.33 \%$ \\
\hline & P2-P3 & $0.19 \%$ & $1.27 \%$ & $7.05 \%$ & $10.45 \%$ \\
\hline \multicolumn{2}{|c|}{ Mean } & $2.16 \%$ & $2.14 \%$ & $19.91 \%$ & $13.66 \%$ \\
\hline \multicolumn{2}{|c|}{ SD } & $2.30 \%$ & $0.98 \%$ & $10.88 \%$ & $2.37 \%$ \\
\hline
\end{tabular}

mimicry is also general mimicry, but not vice versa.

- Mimicry occurs within individual pairs of participants and across all 3 participants simultaneously at different times in the session.

- The increase in specific mimicry in the second half of the session is visible in both groups.

\section{Mimicry During Laughter vs Non-laughter}

Table III shows mean RR and percentage of possible windows where $R R>0$ for specific and general mimicry in groups 1 and 3. Results from the analysis of groups 2 and 4 are not included as they are too low and based on too few instances of mimicry ( $R R>0)$ to be meaningful. Mean RR clearly does not increase during laughter as we might hypothesise from increased affiliation during laughter and predicted from the increased gestures rates during laughter. The frequency of mimicry i.e., the percentage of windows with $R R>0$, appears to be greater in laughter but paired t-tests (2-tailed) do not reach significance (both $p>.1$ ). 
TABLE IV

THE VALUES OF THE AREAS UNDER THE RR'S CURVE IN THE RANGES $[-\tau, 0 s]$ AND $[0 s, \tau]$ WITH $\tau=3 s$ FOR EACH PAIR IN GROUP 1 AND GROUP 3 AND IN LO AND NLO WINDOWS BOTH FOR SPECIFIC MIMICRY (SM) AND GENERAL MIMICRY (GM), RESPECTIVELY.

\begin{tabular}{|c|c|c|c|c|c|c|c|}
\cline { 3 - 7 } \multicolumn{2}{c|}{} & \multicolumn{2}{c|}{ Laughter only (LO) } & \multicolumn{2}{c|}{ Non-laughter only (NLO) } \\
\cline { 2 - 8 } \multicolumn{2}{c|}{} & $\begin{array}{c}\text { AUC } \\
{[-3 s, 0 s]}\end{array}$ & $\begin{array}{c}\text { AUC } \\
{[0 s, 3 s]}\end{array}$ & L/F & $\begin{array}{c}\text { AUC } \\
{[-3 s, 0 s]}\end{array}$ & $\begin{array}{c}\text { AUC } \\
{[0 s, 3 s]}\end{array}$ & L/F \\
\hline \multicolumn{7}{|c|}{ Group 1 } \\
\hline \multirow{3}{*}{ SM } & P1-P2 & 7.50 & 9.35 & 1.25 & 26.0 & 3.22 & 1.24 \\
& P1-P3 & 4.30 & 2.36 & 0.55 & 6.35 & 4.79 & 0.75 \\
& P2-P3 & 9.64 & 1.27 & 0.13 & 20.5 & 21.3 & 1.04 \\
\hline \multirow{6}{*}{ GM } & P1-P2 & 52.9 & 45.8 & 0.86 & 10.7 & 78.9 & 0.74 \\
& P1-P3 & 15.9 & 24.2 & 1.52 & 56.8 & 72.6 & 1.28 \\
& P2-P3 & 22.7 & 18.7 & 0.82 & 88.7 & 79.0 & 0.89 \\
\hline \multicolumn{7}{|c|}{ Group 3 } \\
\hline \multirow{3}{*}{ GM } & P1-P2 & 1.28 & 3.49 & 2.73 & 1.01 & 13.6 & 1.34 \\
& P1-P3 & 4.26 & 4.60 & 1.08 & 1.44 & 2.31 & 1.60 \\
& P2-P3 & 0.46 & 0 & 0 & 7.87 & 1.46 & 0.18 \\
\hline & P1-P2 & 2.41 & 5.61 & 2.32 & 43.5 & 43.1 & 0.99 \\
& P1-P3 & 15.3 & 15.7 & 1.03 & 32.1 & 38.3 & 1.19 \\
& P2-P3 & 12.3 & 3.43 & 2.79 & 38.6 & 36.18 & 0.94 \\
\hline
\end{tabular}

This may be due to the nature of the recording sessions: during all laughter periods, some form of interaction was taking place between participants, since laughter in this context was prompted by the game, chatting or a shared stimulus. Non-laughter periods may have contained phases during which participants were not interacting at all. During such phases, mimicry would be very unlikely as no effective interparticipant interaction was taking place to drive it, and so these phases could legitimately be excluded from analyses. The effect of including any such phases is likely to be small, however, since game rounds, during which interaction was occurring, accounted for $64.6 \%$ and $72.5 \%$ of the recorded session time for groups 1 and 3 respectively. Waiting periods between rounds were relatively short: mean $=47.6 \mathrm{~s}$ and $45.0 \mathrm{~s}$ for groups 1 and 3 respectively. Nevertheless, limiting nonlaughter windows to those containing effective interaction would likely diminish any difference between the LO and NLO results.

\section{Leader-Follower Relationship in Mimicry}

Following the computation of the metric reported in Section $\mathrm{V}-\mathrm{C}$, Table IV reports the areas under the RR curve (AUC) in the ranges $[-3 s, 0 s]$ and $[0 s, 3 s]$ for each pair. They measure to which extent the participants lead and follow each other, respectively, with the ratio between the two, i.e., $L / F=A U C([0,3 s]) / A U C([-3 s, 0 s])$, providing a summary statistic. Figure 2 and Figure 3 show these profiles for group 3 for general and specific mimicry, respectively. These data allow several observations:

- as with the analysis of recurrence across the entire session, RRs for general mimicry are inevitably and substantially larger than RRs for specific mimicry;

- for 5 pairs the leader-follower relationship as defined by the $\mathrm{L} / \mathrm{F}$ ratio is maintained between laughter and nonlaughter periods i.e., both ratios are larger than 1 or both ratios are smaller than 1 . The exception is group $1 \mathrm{P} 2-\mathrm{P} 3$;

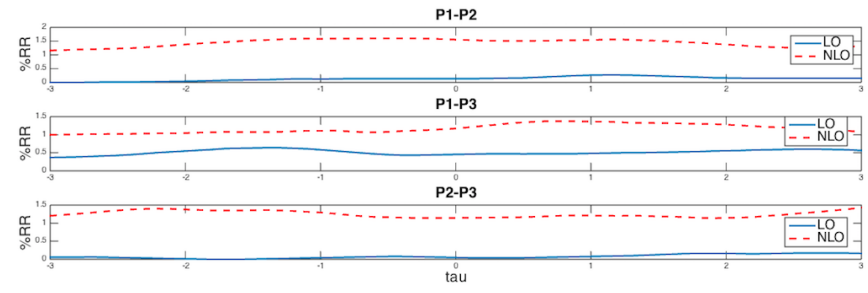

Fig. 2. LO and NLO RR's profile of all pairs in group 3 for general mimicry

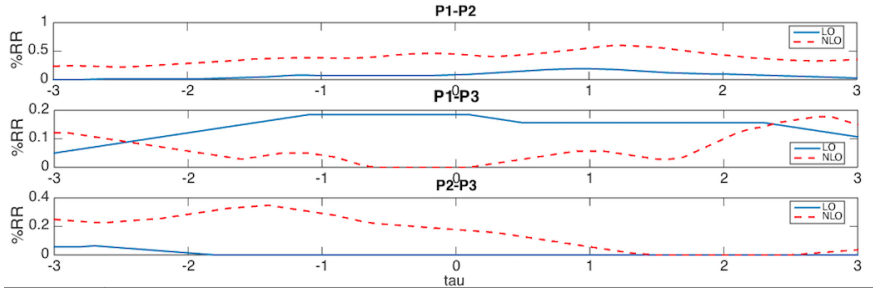

Fig. 3. LO and NLO RR's profile of all pairs in group 3 for specific mimicry

- for general mimicry, the differences between laughter and non-laughter are more varied.

\section{DISCUSSION AND CONCLUSIONS}

Although laughter, as an affiliative signal, was hypothesised to generate higher levels of mimicry we do not observe this. This is especially surprising given that gesture rates are higher during laughter so, even if gesturing is random, mimicry would be expected to increase during laughter. One explanation is that the laughter we observed is just the outward expression of joy in a situation in which strong group affiliations already existed. We also find no evidence that gesture rate during laughter can be predicted from personality-, laughter- or mood-related questionnaires. This reduces the possibility of optimising the affiliative nature of avatar gesture behaviour i.e., mimicry, on the basis of pre-interaction assessment of users; however, gesture rate during laughter can be predicted from gesture rate during non-laughter. Despite the limited similarities between the personalities of participants within group, gesture rates are also strongly related to the group i.e., the gesture rate of any one participant can be reasonably well predicted from the gesture rates of the other participants. A possible explanation is that similar gesture rates within group reflect the overall rapport and enjoyment in each group's session. If a good atmosphere was developed it may have led to greater rates of gesturing in all participants. Our finding that mimicry levels increase over the course of the sessions indicates that, even within groups of friends, familiarity and comfort in a particular scenario may increase emotional contagion. This may be a valuable insight for avatar behaviour in that excessive mimicry too early in an interaction could be seen as inappropriate.

We acknowledge that this study has limitations. The sample size was small, which limits the generalisability of results. Similarly, using groups of friends is a specific situation; however, we felt it was a good initial scenario as it maximises the frequency of laughter [27]. 


\section{REFERENCES}

[1] U. Hess and A. Fischer, "Emotional mimicry as social regulation," Personality and Social Psychology Review, vol. 17, pp. 142-157, 2013.

[2] —_, "Emotional mimicry: Why and when we mimic emotions." Social and Personality Psychology Compass, vol. 8, pp. 45-57, 2014.

[3] C. Frith and D. Wolpert, The Neuroscience of social Interaction. Decoding, Imitating, and Influencing the Actions of Other. Oxford University Press, 2003.

[4] M. Iacobini, T. Gonsalves, N. Bianchi-Berthouze, and C. D. Frith, "Creating emotional communication with interactive artwork," in Affective Computing and Intelligent Interaction, Third International Conference and Workshops, ACII 2009, 2009, pp. 1-6.

[5] A. Wright, E. Shinkle, and A. Linney, "Alter ego. computer reflections of human emotions," in Digital Art, Sixth International Conference, DAC 2005, 2005, pp. 191-199.

[6] R. Dunbar, "Mind the gap: Or why humans are not just great apes," in Proceedings of the British Academy, vol. 154, 2008.

[7] G. S. Hall and A. Allin, "The psychology of tickling, laughing, and the comic," Am. J. Psychol., vol. 9, no. 1, pp. 1-41, 1897.

[8] P. Bourgeois and U. Hess, "The impact of social context on mimicry," Biological Psychology, vol. 77, no. 3, pp. 343-352, 2008.

[9] T. L. Chartrand and J. A. Bargh, "The chameleon effect: the perceptionbehavior link and social interaction." Journal of Personality and Social Psychology, vol. 76, no. 6, pp. 893-910, 1999.

[10] J. Lanzetta and B. Englis, "Expectation of cooperation and competition and their effects on observers' vicarious emotional responses," Journal of Personality and Social Psychology, vol. 56, no. 4, pp. 543-554, 1989.

[11] J. Lakin and T. Chartrand, "Using nonconscious behavioral mimicry to create affiliation and rapport," Psychological Science, vol. 14, no. 4, pp. 334-339, 2003.

[12] H. J. Griffin, M. S. H. Aung, B. Romera-Paredes, C. McLoughlin, G. McKeown, W. Curran, and N. Bianchi-Berthouze, "Perception and automatic recognition of laughter from whole-body motion: Continuous and categorical perspectives," IEEE Transactions on Affective Computing, vol. 6, no. 2, pp. 165-178, 2015.

[13] M. Lafrance and M. Broadbent, "Group rapport: Posture sharing as a nonverbal indicator," Group \& Organization Management, vol. 1, no. 3, pp. 328-333, 1976.

[14] M. M. Louwerse, R. Dale, E. G. Bard, and P. Jeuniaux, "Behavior matching in multimodal communication is synchronized," Cognitive Science, vol. 36, pp. 1404-1426, 2012.
[15] K. Shockley, M.-V. Santana, and C. A. Fowler, "Mutual interpersonal postural constraints are involved in cooperative conversation," $J$. Exp. Psychol. Hum. Percept. Perform., vol. 29, no. 2, pp. 326-332, 2003.

[16] W. Ruch, G. Köheler, and C. van Thriel, "To be in good or bad humor: Construction of the state form of the state-trait-cheerfulness-inventory stci," Pers. Individ. Dif., vol. 18, no. 4, pp. 477-491, 1997.

[17] S. Gosling, P. Rentfrow, and W. Swann, "A very brief measure of the bigfive personality domains," Journal of Research in Personality, vol. 37 no. 6, pp. 504-528, 2003.

[18] W. Ruch and R. Proyer, "Extending the study of gelotophobia: On gelotophiles and katagelasticists," Humor, vol. 22, no. 1/2, pp. 183-212, 2009.

[19] A. Paxton and R. Dale, "Argument disrupts interpersonal synchrony," The Quarterly Journal of Experimental Psychology, vol. 66, no. 11, pp. 2092-2102, 2013

[20] D. C. Richardson and R. Dale, "Looking to understand: The coupling between speakers' and listeners' eye movements and its relationship to discourse comprehension," Cognitive Science, vol. 29, pp. 39-54, 2005.

[21] D. C. Richardson, R. Dale, and N. Kirkham, "The art of conversation is coordination: common ground and the coupling of eye movements during dialogue," Psychological Science, vol. 18, pp. 407-413, 2007.

[22] R. Dale, N. Kirkham, and D. Richardson, "The dynamics of reference and shared visual attention," Front. Psychol., vol. 2, no. 355, 2011.

[23] N. Marwan and J. Kurths, "Nonlinear analysis of bivariate data with cross recurrence plots," Physics Letters A, vol. 302, pp. 299-307, 2002.

[24] F. Orsucci, K. Walter, A. Giuliani, C.L.Webber, and J. Zbilut, "Orthographic structuring of human speech and texts: Linguistic application of recurrence quantification analysis," International Journal of Chaos Theory and Applications, vol. 4, no. 2, pp. 80-88, 1978.

[25] M. Coco and R. Dale, "Cross-recurrence quantification analysis of categorical and continuous time series: an r package," Quantitative Psychology and Measurement, vol. 5, 2013.

[26] J. Nadel, Imitation et communication entre jeunes enfants, ser. Croissance de l'enfant. Presses Universitaires de France - PUF, 1986.

[27] G. McKeown, W. Curran, C. McLoughlin, H. Griffin, and N. BianchiBerthouze, "Laughter induction techniques suitable for generating motion capture data of laughter associated body movements," in Proceedings of the 2nd International Workshop on Emotion Representation, Analysis and Synthesis in Continuous Time and Space (EmoSPACE), 2013. 